\title{
Aportaciones desde el feminismo para la formación del profesorado en el uso de los museos como recursos educativos. Un ejemplo de Aprendizaje-Servicio Contributions from feminism to teacher-training in the use of museums as educational resources. A Service-Learning Project
}

Amaia ARRIAGA. Universidad Pública de Navarra (España). amaia.arriaga@unavarra.es

Resumen: El artículo presenta una experiencia de formación de profesorado en la Universidad Pública de Navarra para el uso de los museos y obras de arte como recursos educativos en educación primaria e infantil. Sus objetivos, contenidos y metodologías se diseñan desde un posicionamiento que está atravesado por la teoría feminista pero que se encuentra y coincide con los planteamientos que se defienden desde otras perspectivas relacionadas con la educación, el arte y la educación artística como la museología crítica, las pedagogías críticas, o las concepciones culturalistas y experienciales sobre el arte. En el artículo se definen cuáles son esos planteamientos teóricos para dejar paso, después, a la explicación sobre cómo se implementan en el aula y los resultados que ofrecen.

La colaboración que en ámbito de la transferencia de conocimiento se desarrolla hace años con el Museo de Navarra ha permitido poner en marcha, además, un proyecto de Aprendizaje-Servicio en colaboración con dos escuelas cercanas al Museo. En este proyecto el alumnado de la asignatura "Recursos de las artes para la educación" de los Grados de Maestro en Educación Primaria y Educación Infantil de la Universidad Pública de Navarra diseña propuestas educativas utilizando las obras del Museo de Navarra y las desarrolla en varias sesiones que se llevan a cabo tanto en las aulas de las escuelas colaboradoras y en las salas del Museo.

Palabras clave: Formación de profesorado; Museo; Educación; Feminismo; Arte; Aprendizaje-Servicio.

Abstract: The article presents an experience of teacher training at the Public University of Navarra for the use of museums and works of art as educational resources in primary and early childhood education. Its objectives, contents and methodologies are designed from a position that is influenced by feminist theory but that meets and coincides with the approaches that are defended from other perspectives related to education, art and artistic education such as critical museology, critical pedagogies, or culturalist and experiential conceptions about art. The article defines these theoretical approaches and explains how they are implemented in the classroom and the results they offer. 
The collaboration that in the field of knowledge transfer has been developed for years with the Museum of Navarra has also allowed the launching of a Service-Learning project in collaboration with two schools situated near the Museum. In this project, the students of the course "Resources of the arts for education" of the Degree in Primary Education and Early Childhood Education of the Public University of Navarra design educational proposals using the works of the Museum of Navarra and they develop these proposals in several sessions that are carried out both in the classrooms of the partner schools and in the Museum galleries.

Keywords: Teacher-Training; Museum; Education; Feminism; Art; Service-Learning.

\section{Introducción}

Desde 2016, el grupo de investigación Edarte trabaja en estrecha colaboración con el Museo de Navarra diseñando y elaborando los recursos de interpretación que se están implementando en la renovación de su colección permanente (Arriaga, Aguirre, 2020).

Esta colaboración ha permitido poner en marcha un proyecto de Aprendizaje-Servicio en el que el alumnado de la asignatura "Recursos de las artes para la educación" diseña propuestas educativas utilizando las obras del Museo de Navarra y las desarrolla con dos escuelas colaboradoras en varias sesiones que se llevan a cabo tanto en las aulas de las escuelas y como en las salas del Museo.

La asignatura optativa se ofrece en el tercer curso y la cursan, conjuntamente, el alumnado del Grado en Educación Infantil y el de Educación Primaria de la Universidad Pública de Navarra. Sus objetivos, contenidos y metodologías se diseñan desde un posicionamiento que está atravesado por la teoría feminista pero que se encuentra y coincide, en muchos casos, con los planteamientos que se defienden desde otras perspectivas relacionadas con la educación, el arte y la educación artística. En las siguientes páginas se presentará, primero, cuáles son esos planteamientos teóricos para dejar paso, después, a la explicación sobre cómo se implementan en el aula y los resultados que ofrecen.

\section{Aportaciones de la teoría crítica y feminista al diseño de la asignatura}

\section{Intersección entre museología crítica y pedagogía crítica}

En la base del diseño de esta asignatura está la intersección entre museología crítica (Duncan, 1995; Padró, 2005) y pedagogía crítica (Apple, 1975; Ellsworth, 1989; Giroux, 1990; Gore, 1992), dos corrientes fuertemente influidas por el feminismo y el poscolonialismo y que han abordado las relaciones entre saber y poder en el museo y la escuela. Así, han analizado cómo estas instituciones están atravesadas por ideología y cómo en ellas se manifiesta un determinado orden social, político y económico. 
Basándonos en sus planteamientos, la primera parte de la asignatura busca que los/as futuros maestros/as se planteen por qué y cómo determinados aspectos de la cultura colectiva, vinculados a los discursos hegemónicos, se presentan en la escuela y en el museo como conocimientos objetivos y verdaderos, legitimando, así, los intereses dominantes de la sociedad.

En el caso de los museos, se tienen en cuenta trabajos que han analizado las formas en que las diferencias y, en especial, las desigualdades relacionadas con la raza, el género, la sexualidad y la clase, son reproducidas a través de las exclusiones que produce "el canon" y "lo normativo" (Macdonal, 2011). Así nos son de utilidad los planteamientos de académicas como Carol Duncan (1995) o Margaret Lindauer (2006), por ejemplo.

\section{El arte como sistema cultural y como experiencia}

En la concepción del arte que se maneja en el diseño de la asignatura también tienen peso las perspectivas críticas. Así se plantea un acercamiento al arte entendiéndolo como sistema cultural, enfatizando la vinculación que el hecho artístico tiene con el sistema en el que nace o se consume (Geertz, 1994) y abordándolo como un mediador cultural de representaciones sociales relacionadas con el poder, la religión, las relaciones sociales, la belleza, el cuerpo, etc.

Además, de la mano de John Dewey (1934) y de quien ha aplicado sus ideas a la educación artística (Agirre, 2000) se entiende el arte como experiencia y las obras de arte como acontecimientos capaces de generar o activar experiencias que permiten al alumnado repensarse a uno mismo a través de las imágenes. De acuerdo con Imanol Agirre (2000: 300) se considera que "[...] el interés de la comprensión artística no reside tanto en su eficacia para proporcionar conocimiento sobre el «otro-productor», como en su poder para configurar identidad y desarrollar sensibilidad estética, transformando las experiencias ajenas en tomas de conciencia sobre la propia existencia" y es desde este planteamiento desde el que se diseñan las actividades que se ocupan de trabajar directamente con obras de arte.

\section{La centralidad de la experiencia}

La centralidad la experiencia es una idea que impregna no solo la concepción del arte sino también la de la propia educación. Las pedagogías progresistas hace tiempo que defendieron el valor de la experiencia en la educación porque moviliza afectos, emociones y reflexiones que son fundamentales en la construcción del aprendizaje y de la subjetividad.

En este sentido, John Dewey criticó la tradicional separación entre pensar y hacer, reflexión y acción, mente y cuerpo. Como indican Valmonde y Nubiola (2016), frente a la consideración aislada y abstracta de la razón, interpretó la inteligencia como una función vital cuyo papel consiste en ordenar la experiencia que es, a su vez, la base del pensamiento y denunció la minusvaloración del cuerpo, de la experiencia ordinaria, de las emociones y de la naturaleza, y su separación del espíritu, del orden teórico y de la dirección social. En este sentido, las ideas de Dewey coinciden con las pedagogías feministas y su énfasis en la experiencia corporal, emocional, la subjetividad y los relatos personales. 


\section{Pedagogía feminista y el hacer de la educadora}

Bell Hooks (2003) señaló que la educación como práctica de libertad no debe reducirse a la transmisión de un conocimiento liberador, esto es, al empleo de materiales diferentes o más radicales, sino que debe desarrollar una práctica liberadora en clase. En este sentido defendió que una acción como la inclusión de la experiencia personal en el aula podía suponer un desafío mucho más constructivo que el simple hecho de cambiar el programa de estudios.

Desde esta perspectiva, la reflexión en esta asignatura se dirige a cuestiones apuntadas desde las teorías críticas, pero siempre impregnadas por unas formas de hacer que tienen mucho más en cuenta las experiencias personales, la negociación y el co-aprendizaje y que favorecen unas relaciones menos jerarquizadas y más horizontales.

Otra de las reivindicaciones de las pedagogías feministas tiene que ver con la valorización del cuidado, que se traduce en esta asignatura en el intento de garantizar que el aula sea un espacio de seguridad para el alumnado, donde sienta que se puede desarrollar el diálogo y la negociación.

Como se ha comentado, desde esta corriente toma importancia la implicación del cuerpo, los afectos y las emociones en los procesos aprendizaje. Ahmed (2004) y Boler (1998) señalan que la experiencia emocional y afectiva encarnada es fundamental en las acciones educativas porque tienen un valor incontestable en los procesos de construcción de la identidad.

De alguna manera, las pedagogías feministas se encuentran aquí con la crítica que desde otras instancias y miradas se ha hecho a la tendencia de la educación formal hacia el razonamiento lógico-matemático o el desarrollo de la lectoescritura, dejando de lado otro tipo de inteligencias (Gardner, 1983), otras formas de conocimiento e interacción con la realidad en la que intervienen otras dimensiones y capacidades humanas, muchas de ellas relacionadas con el actuar del cuerpo y las emociones.

\section{De la teoría a la práctica. Propuestas y actividades en el aula}

¿En qué tipo de propuestas y actividades se materializan estos principios y concepciones sobre los museos, el arte, la educación y la educación artística? En las siguientes líneas se explica cómo se va desarrollando la asignatura, el tipo de ejercicios y actividades que se realizan y en qué trabajos se concretan, intercalando ejemplos de algunos de los realizados por el alumnado.

\section{Conociendo, críticamente, los museos de nuestro entorno}

Las primeras semanas de la asignatura las dedicamos a visitar los museos de nuestro entorno y tratar de convertirnos en lo que Margaret Lindauer (2006) llamó "visitantes críticos". 
Para ello tomamos de Carol Duncan (1995) el análisis de los museos como "estructuras rituales", construcciones simbólicas y sociales en las que se satisfacen o afirman determinadas creencias y valores, identidades de clase, políticas o sexuales, mediante la dramatización de sus formas y espacios arquitectónicos, itinerarios y narraciones históricoartísticas.

\section{El contenedor. Escenario para un drama}

La académica estadounidense propone pensar el espacio del museo como el escenario de un drama, un escenario que invita a los/as visitantes a interpretar una determinada representación (ritual). Así, visitamos la Fundación Museo Jorge Oteiza para analizar cómo el edificio y sus diferentes espacios son determinantes en nuestra experiencia, son elementos que modelan nuestro encuentro con los objetos u obras de arte e influyen en nuestra forma de pensarlos.

Como propone Aída Sánchez de Serdio, abordamos el espacio construido del museo como interlocutor, es decir "una instancia que entra en un diálogo activo con los sujetos, artefactos, discursos, prácticas, cuerpos y afectos que lo transitan y habitan" (S. de Serdio, 2017, p.1), entendiendo que la arquitectura conforma mundos físicos, mentales y emocionales.

Para hacer este tipo de análisis se ofrece al alumnado una guía para la reflexión con varias preguntas que les invitan a sentir y vivir el espacio, a ser conscientes de su transitar por él. Este acercamiento guiado da pie a reflexiones como la que realiza este alumno del curso 2018-2019 sobre la Fundación Museo Jorge Oteiza:

Altzuza no es un lugar de paso. No hay transporte público, se trata de uno de esos pequeños pueblos en los que se acaba la carretera. Hasta aqui no llega el sonido de las sirenas, portador de malos augurios. Parece que el lugar que eligió Jorge Oteiza para su retiro nos incita, nos anima a iniciar nuestro propio retiro. (...) .

Como recuerda Duncan (2007) a los museos se accede por grandes escalinatas, y este hecho debe considerarse dentro de la experiencia museística como parte de nuestra visita.

En el caso del Museo de Oteiza es notable el efecto que provoca la ascensión continuada, casi sin fin, hasta llegar a la puerta. Esa situación elevada parece prometer un encuentro distinto. "El espacio que ocupa el museo ha sido cuidadosamente diferenciado y reservado para recibir una atención muy especial" (Duncan, 2007, p.26).

O como la que realiza otra alumna del curso 2019-2020:

Bajamos el volumen de voz en cuanto entramos, llegando casi a susurrar, sin que nadie nos obligara a ello. Era el entorno en el que nos encontrábamos lo que hizo que nuestro comportamiento cambiara en cierto modo. De acuerdo con Jorge Luis Marzo: Estas instituciones se modelaron como espacios públicos en los que exponer el grado de éxito conseguido a la hora de disciplinar la propia privacidad, de amaestrar la propia individualidad, de reprimir las emociones: "Entre en el museo como visitante, salga como ciudadano" (Marzo, J.L., 2013)". 


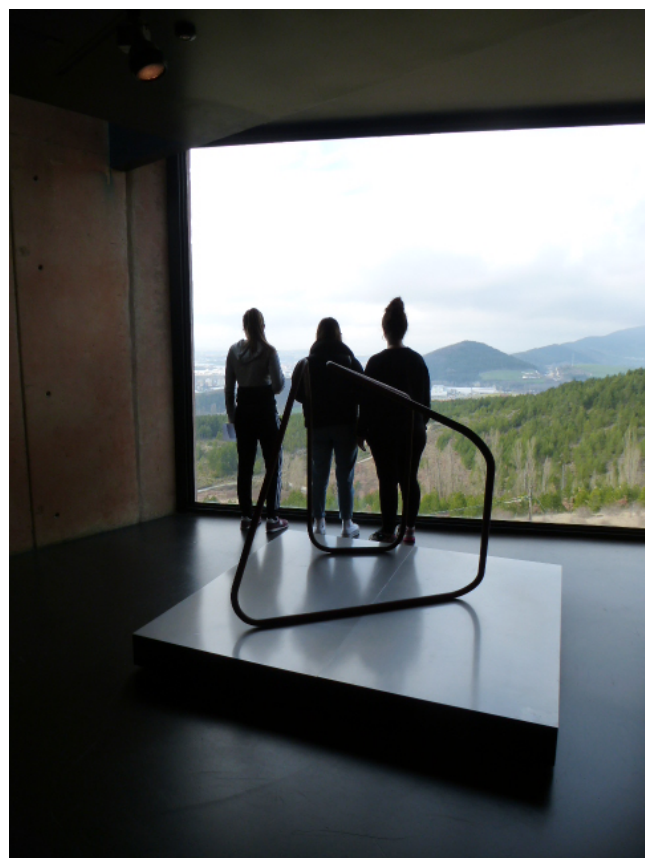

Figura. 1. Visita a la Fundación Museo Jorge Oteiza con el alumnado del Grado de Maestro. Fotografía de la autora

\section{Las poéticas y políticas de la selección y exposición}

En el texto mencionado anteriormente, Margaret Lindauer (2006) propone analizar los objetivos declarados y no declarados del museo, sus contradicciones y este es otro de los objetivos que abordamos en estas visitas. Para ello nos es de utilidad el concepto de currículum oculto, acuñado por Phillip W. Jackson en 1968 y que ha servido habitualmente para analizar los contenidos de la educación formal. En nuestro caso esta herramienta nos permite examinar cómo normas, valores y creencias que no son afirmadas explícitamente por museos o centros de arte a menudo contradicen los objetivos explícitos de estas instituciones y son transmitidas a los/as visitantes, a través de elementos o detalles aparentemente neutrales como la arquitectura de los edificios, la clasificación y la yuxtaposición de objetos en una exposición, el uso de vitrinas o dispositivos interactivos, etc. (Macdonal, 1998).

Con estas herramientas en mente, visitamos el Museo de Navarra, el museo de arte y patrimonio histórico más importante de nuestra comunidad, porque supone un espacio ideal para reflexionar sobre las políticas adquisición y exposición de obras y los intereses e ideologías que las han sustentado.

Para desvelar “...qué discursos circulan y se promueven, cómo y por qué, qué se ignora o qué se da por sentado y no se pone en cuestión" (Macdonald, 2011: 3) tan importante es lo que está como lo que no está. Así, por ejemplo, en ese recorrido por el Museo de Navarra hay quien denuncia la ausencia de mujeres artistas, hay quien echa en falta obras y objetos vinculados al carlismo o a un alumno puede llamarle la atención que un retrato de Jose 
María de Huarte y Jauregui, pintado por Ignacio Zuloaga esté fechado en plena guerra civil y empiece a investigar para conectar la historia del Museo de Navarra, la del Conde de Rodezno, figura preeminente del franquismo y la historia de la comunidad foral:

Una de las obras que llama mi atención durante la visita es el retrato de José María de Huarte y Jáuregui de Ignacio Zuloaga. Es un lienzo de tamaño imponente, en el que queda de manifiesto la pericia técnica del autor. En mi opinión es una de esas obras cuyo valor estético resulta prácticamente indiscutible. Después de este primer acercamiento a la obra, reparo en la fecha en la que se realizó. La fecha es 1937. Este dato me pone en alerta y empiezo a preguntarme qué hay detrás de una obra realizada en plena guerra civil. (...) La obra de Zuloaga fue adquirida por el Museo de Navarra en 2007, 55 años después de la muerte del Conde de Rodezno. (Trabajo alumno 2018/2019)

La teoría que trabajamos en clase, con referentes como Carol Duncan, Sharon MacDonald, Alan Wallach o Margaret Lindauer ayuda al alumnado a realizar este tipo de reflexiones, pero también se aportan referentes del mundo de la creación artística, específicamente de artistas que realizan crítica institucional como como Andrea Fraser o Fred Wilson. Por ejemplo, el alumnado debe basarse en la obra "Mining the museum" de este último para hacer una propuesta de intervención en el Museo de Navarra que ayude a desvelar y confrontar su currículum oculto. Así, ese mismo alumno que se fija en el cuadro de Ignacio Zuloaga propone colocarlo junto a la obra de Abel Azcona (2015) "Enterrados" que se realizó, justamente, en la plaza Conde Rodezno, frente al Monumento a los Caídos, con el objetivo de reivindicar la memoria histórica.

También se ofrecen ejemplos de la historia de las exposiciones que nos ayudan a pensar cómo la forma de clasificar, ordenar y presentar las obras seleccionadas también influye en nuestro encuentro con ellas. Por ejemplo, la exposición "ART/artifact" (1988), comisariada por Susan Vogel, nos permite entender que los métodos de exposición imponen significados culturales, sociales o históricos a los objetos e influyen en nuestra percepción, interpretación y valoración de los mismos.

\section{Museos y educación. Tendencias educativas y concepciones de arte e interpretación}

Aunque la reflexión sobre la función educativa del museo está presente desde el inicio de la asignatura, el segundo bloque temático enfoca directamente sobre cuestiones educativas en el contexto de la colaboración entre museos y escuelas.

En esta fase, de nuevo, se tiene en cuenta la teoría que se ha construido desde perspectivas críticas y feministas. Primero, se presentan las diferentes narrativas museísticas y tendencias en educación que se pueden encontrar en museos y centros de arte, utilizando para ello la categorización que hizo Carla Padró (2005) y la relación de esas categorías con contextos históricos e ideológicos concretos (Arriaga, 2011).

En cada una de estas narrativas y tendencias se marcan los objetivos, contenidos y metodologías que se ponen en funcionamiento, así como los roles que educadores/as, profesores/as y alumnos/as toman en el proceso de enseñanza-aprendizaje y, especialmente, en el desarrollo de procesos de interpretación o acercamiento a las obras de arte. 
Para incidir en esta última cuestión, trabajamos las concepciones de arte e interpretación que subyacen a las diferentes formas de trabajar lo educativo en el museo, utilizando, junto con ejemplos ilustrativos, la clasificación que presenta cuatro grandes maneras de concebir el arte y la interpretación que se pueden dar, y habitualmente se dan, en contextos educativos (Arriaga, 2009, 2010).

\section{Análisis de recursos y actividades educativas ofrecidas por museos}

Estas dos categorizaciones se utilizan para analizar recursos didácticos como cuadernillos y actividades educativas que educadoras de museos de la comarca de Iruñea-Pamplona desarrollan con alumnado de infantil y primaria.

En el caso de los recursos escritos, entre la selección, siempre se ofrecen algunos que se hayan diseñado aplicando una mirada de género, como las guías didácticas (Fernández; López Fernández Cao y Bernárdez, 2013) del proyecto Museos en Femenino dirigido por Marian López Fernández Cao.

De la aplicación de la teoría al análisis de recursos emergen reflexiones como la que hace este alumno del curso 2018/2019) sobre un cuadernillo didáctico del Museo Oteiza:

Los principales contenidos presentados son la propia obra del artista y su presentación y explicación a través de un mediador/a experta que nos ayuda a descodificar el mensaje que esconde la obra escultórica del artista. Se trata de un planteamiento ligado al rol estético e instructivo-disciplinario omnipresente en la historia de los museos. Se nos presenta el contenido del objeto (obra) como una realidad objetiva que se vincula con una única y correcta explicación-interpretación. En todo caso, son contenidos vinculados a la idea de genio-artista, en los que se puede vislumbrar cierto aroma mesiánico por parte de la institución. No se plantean preguntas al alumnado. Únicamente se propone un debate en torno a las formas esféricas que podemos encontrar en la naturaleza y su relación con el vacío. No obstante, y al hilo de ello, parecen coherentes las actividades que luego se desarrollaran, puesto que inciden en la misma temática. En las actividades propuestas, una vez terminada la visita, parece, por un lado, abordarse un proceso de imitación de la obra del artista, dando instrucciones precisas sobre el modo en que esta imitación podría realizarse. Por otro lado, se intuye un intento de acercamiento a lo personal(alumnado) con expresiones como "disfrutar de tu propio universo", aunque esto último no termine de encajar del todo con el tono general de la oferta educativa del museo. (Trabajo alumno 2018/2019)

\section{Estrategias didácticas acercamiento obras}

Llegados a este punto, el alumnado conoce los museos y su oferta educativa y tiene un posicionamiento crítico frente a estos dos elementos. Es el momento de trabajar estrategias didácticas para poder poner en marcha un acercamiento a las obras de arte que coincida con los principios educativos y estéticos que están en la base de la asignatura y que serán, también, el lugar desde el que el alumnado tendrá que diseñar sus propuestas educativas.

Una primera herramienta que nos es de utilidad es la propuesta Ways In de las galerías Tate en la que proponen cuatro enfoques desde los que interpretar las obras de arte (Charman, 
Rose y Wilson, 2006) y que nos permite ser conscientes de las prácticas de interpretación que ponemos en marcha y planificar las que nos gustaría fomentar.

\section{La experiencia personal como punto de partida para la interpretación}

La propuesta Ways In da mucha importancia al enfoque personal a la hora de interpretar el arte, y coincide así con el planteamiento de que, en contextos educativos, la fuente principal para la construcción del significado frente a las obras de arte debe ser la experiencia personal del alumnado. Desde este punto de vista, en las actividades y ejercicios que se realizan en clase se apela a la experiencia vital, anímica y cultural de los/as alumnos/as como punto de partida de la interpretación de las obras de arte. Se coincide así con los postulados de Dewey y del feminismo y su interés por romper con las tradicionales jerarquías de saber y la diferenciación entre expertos/as e ignorantes. Como señaló Carla Padró (2005, p. 150) la interpretación no es de "aquellos que poseen un conocimiento especial, sino de aquellos que tienen conocimiento".

\section{El contexto de producción y de uso en la interpretación de las obras de arte}

Concebir el arte desde un enfoque culturalista, obliga, además, a dar protagonismo a los contextos culturales y a los usos del arte en esos procesos de construcción de significado en los que se sumerge el alumnado en el aula.

Barthes señala (1967/1977, p. 26): “...leer fielmente depende de mi cultura, de mi conocimiento del mundo..." y las corrientes feministas y postcolonialistas añaden que la diferencia cultural está marcada por diferencias de clase, raza, género, generación o sexualidad. Esta es una cuestión que se tiene en cuenta también a la hora de proponer enfoques desde los que interpretar las obras de arte en el marco de esta asignatura (Pollock, 1988, Mayayo, 2003).

De acuerdo a estas perspectivas críticas, las cuestiones vinculadas a la raza, el sexo y la clase toman relevancia en la forma en que se aborda la cuestión de la interpretación de las obras de arte en el aula.

\section{La producción estética como medio para la interpretación}

En relación a las prácticas de interpretación de las obras de arte, tratamos de alejarnos también de la tendencia de la educación artística postmoderna a canalizar las actividades de análisis e interpretación estética a través de la elaboración de textos críticos o debates, perpetuando "la tradicional separación entre la respuesta estética, dependiente de lo verbal y la actividad creadora, que usa los instrumentos expresivos propios del medio (pictórico, infográfico, escultórico...)" (Aguirre, 2005, p.19). Nuestra intención es soslayar, en esta asignatura, la vieja distinción entre comprensión y producción y restaurar el equilibro deseable entre ambas dimensiones de la actividad formativa en educación artística. Así se ponen en práctica estrategias de interpretación de las obras de arte que se desarrollan y materializan a través de producciones literarias, rítmicas, performances o instalaciones, 
como las que Imanol Aguirre denominó "producciones comprensivas”. Esta estrategia promueve "la capacidad para sensibilizar y crear narrativas sobre los productos estéticos, (en forma de) elocuentes artefactos estéticos." (Aguirre, 2005, p. 19). Y facilita, además, un acercamiento multisensorial (no sólo visual) a las obras de arte y en el que el cuerpo pueda tomar mucho más protagonismo.

\section{Diseño de propuestas educativas en el marco del aprendizaje-servicio}

Uno de los objetivos a la hora de diseñar la asignatura ha sido desarrollar procesos que permitan un aprendizaje más significativo y motivador. Por esta razón, como se ha explicado en otros textos (Arriaga, 2015) desde que se empezó a impartirla, buscamos proponer actividades que se acercaran al método por proyectos que, como indica Díez $(1995, \mathrm{p} .33)$ "trata de llevar a la escuela (en este caso universidad) el modo natural de aprender que tenemos los seres humanos" y que estos proyectos tuvieran una materialización en el "mundo real", fuera de los espacios y tiempos del aula universitaria.

La colaboración en el ámbito de la transferencia de conocimiento que desarrollamos desde 2016 con el Museo de Navarra, nos permitió, en 2017-18, poner en marcha una colaboración con dos escuelas cercanas al Museo (Escuela Pública San Francisco y Colegio Santo Tomás-Dominicas), para que los proyectos que el alumnado elabora pudieran desarrollarse con niños y niñas de educación infantil y primaria en las aulas de esos centros y en las salas del propio Museo.

Estos proyectos se convierten en el trabajo final de la asignatura que supone un tema del programa en sí mismo. En él trabajamos cuestiones prácticas sobre el desarrollo de propuestas educativas que utilicen como recurso museos y obras de arte, pero recogiendo y aplicando los aprendizajes desarrollados en los anteriores bloques del temario.

Así, de la misma manera que la asignatura se diseña desde una idea de arte como sistema cultural y una idea de arte como experiencia, las propuestas educativas que el alumnado plantea también deben enfocarse desde esta perspectiva. En sus proyectos el arte debe convertirse en una herramienta para la interpretación de la cultura y la construcción de la subjetividad de los niños y niñas, y las obras de arte en detonantes que les permitan enriquecer sus experiencias vitales, pensarse y pensar su mundo redescubriéndose/lo a la luz de los productos de otros/as.

Dentro de este marco, el alumnado de la asignatura contacta y trabaja con el profesorado de las escuelas colaboradoras para buscar temas interesantes que puedan trabajarse a través de las obras del Museo de Navarra. Así, se consensua un hilo temático para la propuesta educativa que debe conectar con cuestiones que afectan a esos niños y niñas como pueden ser la construcción de la identidad, la emigración, la relación del ser humano con la naturaleza, la muerte, etc.

Estas propuestas son llevadas a la práctica en 3 sesiones. La introductoria se realiza en la escuela, la segunda en las salas del Museo de Navarra y la tercera, de nuevo, en la escuela, 
para dar conclusión al mini-proyecto a través de una actividad creativa que tiene como objetivo recoger o volcar el aprendizaje que se ha dado a lo largo del proceso.

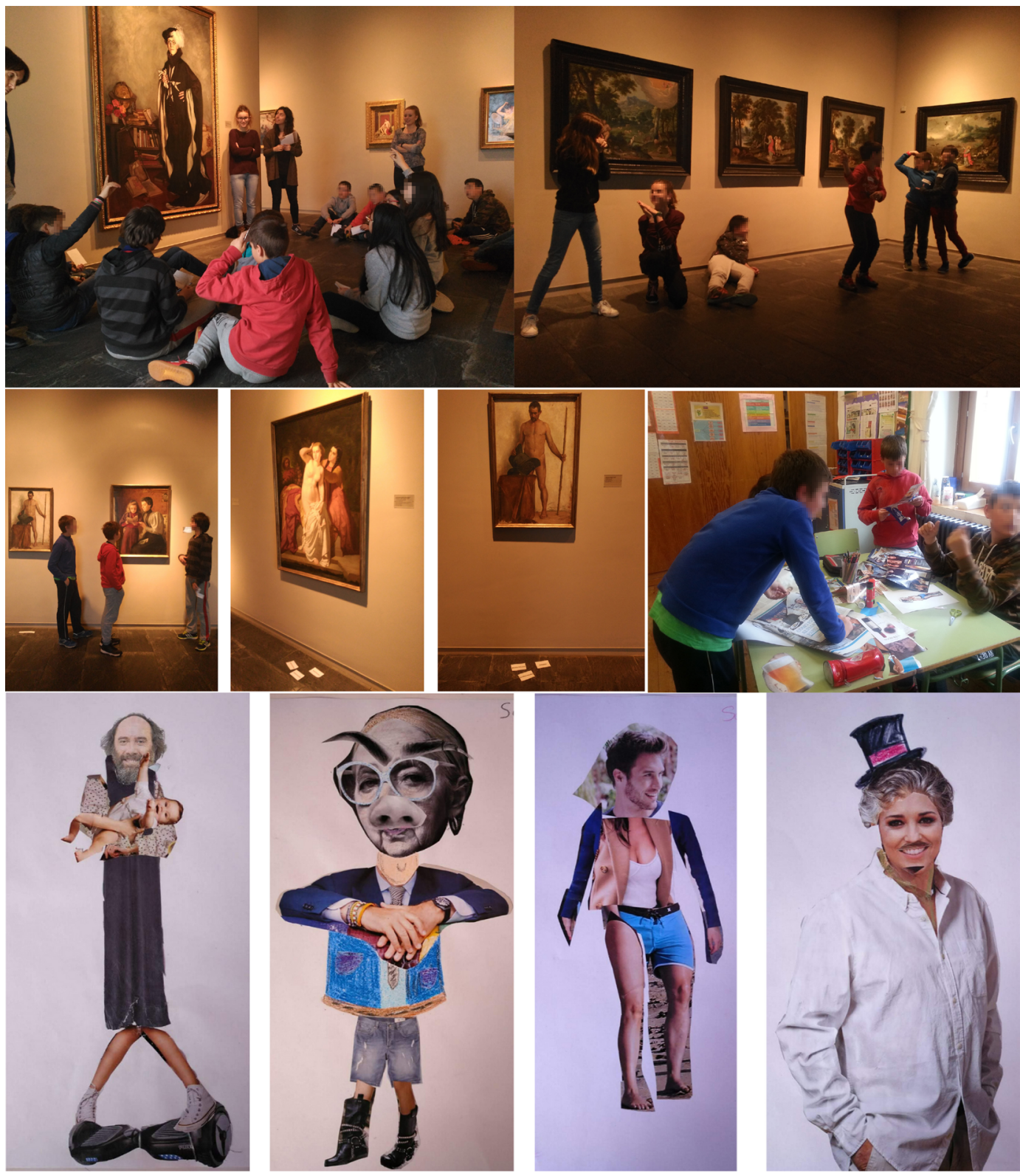

Figura 2. Actividades del proyecto sobre masculinidades “¿Qué es ser chico?” desarrollado por las alumnas Ane Villanueva, Irati Ornat y Uxue Pérez

Plantear proyectos con fundamento en el mundo real permite generar experiencias reales para los/as estudiantes que favorecen la adquisición de habilidades, capacidades y estrategias tan necesarias para ejercer su futura profesión. De esta manera la asignatura se acerca, además, a las propuestas de la metodología de "Aprendizaje Servicio", que propone combinar el conocimiento en las aulas con la participación de los/as alumnos/as 
en proyectos de prestación de servicios gratuitos a la sociedad a través de colaboraciones con instituciones y entidades sin ánimo de lucro.

Las investigaciones internacionales sobre el APS han revelado impactos positivos en seis áreas de desarrollo del alumnado: académico y cognitivo, cívico, vocacional profesional, social, personal, ético y moral (Conwway, Amel y Gerwien, 2009; Eyler, Stenson y Gray, 2001; Furco, 2003; Yorio y Ye, 2012). Además, la metodología del "aprendizaje-servicio" es un enfoque más que interesante, porque recoge muchas de las aspiraciones que se han defendido en anteriores apartados. Es una propuesta totalmente vinculada a la filosofía de la educación experiencial que replantea nuestro rol como profesores/as dejando el protagonismo pedagógico de la experiencia al estudiante que aprende haciendo. También coincide con las aspiraciones de la pedagogía crítica, por su vocación social, solidaria y transformadora y por su aproximación ética a la práctica docente. Además, como señaló Nieves Ledesma en el XII Encuentro Estatal de Aprendizaje-Servicio, celebrado en Pamplona en noviembre del 2019, en todas las experiencias de Aprendizaje y Servicio subyace una mirada feminista, porque enlazan saber y vida, se centran en el cuidado y atienden a necesidades sociales, medioambientales, etc.

En todo caso, no es un trabajo carente de dificultades, exige mucha coordinación y hace aflorar resistencias e inercias en la institución universitaria. Pero esperamos que eso mismo sea un signo de que nos estamos aventurando en la buena dirección.

\section{Referencias bibliográficas}

Agirre, I. (2000). Teorías y prácticas en educación artística. Universidad Pública de Navarra.

Aguirre, I. (2005). Educar desde y para la experiencia estética. La educación artística y la formación de los sujetos. En F. Miranda y G. Vicci (Compl.), La Educación artística preuniversitaria (pp. 3-23). UDELAR.

Ahmed, S. (2004). Feminist attachments. En S. Ahmed (Ed.). The Cultural Politics of Emotion (pp. 168-190). Edinburgh University Press.

Apple, M. W. (1975). Curriculum as Ideological Selection. Comparative Education Review, 20, 209-215.

Arriaga, A. (2009). Conceptions on art and interpretation in the educational discourses and practices at Tate Britan [Tesis doctoral, Universidad Pública de Navarra].

Arriaga, A. (2011). Desarrollo del rol educativo del museo: narrativas y tendencias educativas. Revista Digital do LAV, 7(4), 1-23.

Arriaga, A. (2015). Formación de profesorado para la utilización de centros de arte como recursos para la educación infantil y primaria. Matéria-Prima. 3(2), 131-141.

Arriaga, A. y Aguirre, I. (2010). Un aparato metodológico para analizar las ideas de arte e interpretación que subyacen en discursos y prácticas educativas de museos de arte. Revista 
Iberoamericana de Educación, 53, 203-223.

Arriaga, A.; Aguirre, I. (2020). Museum-university collaboration to renew mediation in art and historical heritage. The case of the Museo de Navarra. Arte, Individuo y Sociedad, 32 (4), 989-1008.

Bernárdez, A.; López Fernández Cao, M.; Fernández Valencia, A. \& Beteta, Y. (2013). Guía didáctica Museo Reina Sofía. Museo Reina Sofía.

Boler, M. (1998). Feeling power: Emotions and education. Routledge.

Charman, H., Rose, K. y Wilson G. (Eds.). (2006). The art gallery handbook: a resource for teachers. Tate Publishing.

Conway, J. \& Amel, E. \& Gerwien, D. (2009). Teaching and Learning in the Social Context: A Meta-Analysis of Service Learning's Effects on Academic, Personal, Social, and Citizenship Outcomes. Teaching of Psychology, 36, 233-245. doi:10.1080/00986280903172969

Dewey, J. (1934). Art as Experience. En Jo Ann Boydston (Ed.) (1967) Later Works of John Dewey. (Vol. 10). Southern Illinois University Press.

Diez Navarro, C. M. (1995). La oreja verde de la escuela. Trabajo por proyectos y vida cotidiana en la escuela infantil. Ediciones De la Torre.

Duncan, C. (1995). Civilizing rituals inside public art museums. Routledge

Ellsworth, E. (1989). Why Doesn't This Feel Empowering? Working Through the Repressive Myths of Critical Pedagogy. Harvard Educational Review. 59(3), 297-325.

Eyler, J., Stenson, C.M., \& Gray, C.J. (2001). At A Glance: What We Know about The Effects of Service-Learning on College Students, Faculty, Institutions and Communities, 1993- 2000. https://digitalcommons.unomaha.edu/slcehighered/139.

Fernández, A.; López Fernández Cao, M. y Bernárdez, A. (2013). Guía didáctica Museo Reina Sofia. Museo Reina Sofía.

Furco, A. (2003). El impacto educacional del aprendizaje-servicio. ¿Qué sabemos a partir de la investigación? [Diapositivas de PowerPoint] University of California-Berkeley. http://roserbatlle.files.wordpress.

Gardner, H. (1983). Frames of mind: The theory of multiple intelligences. Basic Books.

Geertz, C. (1994). Conocimiento local. Paidós.

Giroux, H. (1990). Los profesores como intelectuales. Paidós Ibérica

Gore, J. M. (1992). What We Can Do for You! What Can "We" Do for "You"? Struggling over Empowerment in Critical and Feminist Pedagogy. En C. Luke, y J. Gore (Eds.), Feminisms and Critical Pedagogy (pp. 54-73). Routledge.

Hooks, b. (2003). Teaching Community, A Pedagogy of Hope. Routledge. 
ARRIAGA / Aportaciones desde el feminismo para la formación del profesorado en el uso de los museos como recursos educativos. Un ejemplo de Aprendizaje-Servicio. Dossier EARI 2021

Lindauer, M. I. (2006). The critical museum visitor En J. Marstine (Ed.). New Museum Theory and Practice. An introduction (pp. 203-225). Blackwell Publisher.

Macdonal, S. (1998). The politics of Display: Museums, Science, Culture. Routledge. doi: $10.4324 / 9780203838600$

Macdonal, S. (2011). Expanding Museum Studies: An Introduction. En S. Macdonal. (Ed.). A Companion to Museum Studies (pp.14-16). Whiley-Blackwell.

Marzo, J.L. (23 de enero de 2013). Cómo mirar el museo. La vanguardia.com. http://www. lavanguardia.com/cultura/20130123/54363007231/como-mirar-el-museo.htm

Mayayo, P. (2003) Historia de mujeres, historia del arte. Cátedra.

Padró, C. (2005). Educación artística en museos y centros de arte. En R. Huerta y R. De La Calle (Eds.), La mirada inquieta. Educación artística y museos (pp. 137-152). PUV.

Pollock, G. (1988). Vision and Difference: Feminity, Feminism, and the Histories of Art. Routledge.

Sánchez de Serdio, A. (2017) Prácticas, discursos, cuerpos y espacios. Diálogos críticos entre arquitecturas, pedagogía y política en la educación en museos. En R. Huerta y A. Alonso-Sanz (Eds.) Entornos informales para educar en artes. (pp. 77-88). Publicacions de la Universitat de València.

Vaamonde Gamo, M., \& Nubiola, J. (2016). El legado feminista de John Dewey. Espacio, Tiempo y Educación, 3(2), 281-300. doi: 10.14516/ete.2016.003.002.012

Yorio, P.L. y Ye, F. (2012). A meta-analysis on the effects of Service-Learning on the social, personal, and cognitive outcomes of learning. Academy of Management, Learning \& Education, 11(1), 9-27. doi: 10.5465/amle.2010.0072 